be rare (e.g. Yoruba, Met frequency 0.0004 in a group of 226 sampled; African Americans 0.05 in a group of 90) so that a genetic association analysis with depression would require very large samples.

Although we would anticipate the functional impact of being a Met carrier to be similar across population groups, we agree with Yeebo that this would warrant further investigation.

1 Verhagen $M$, van der Meij $A$, van Deurzen PA, Janzing JG, Arias-Vásquez A Buitelaar JK, et al. Meta-analysis of the BDNF Val66Met polymorphism in major depressive disorder: effects of gender and ethnicity. Mol Psychiatry 2010; 15: 260-71.

Cynthia H.Y. Fu, School of Psychology, University of East London, Stratford Campus, Water Lane, London E15 4LZ, UK. Email: c.fu@uel.ac.uk, and Centre for Affective Disorders, Institute of Psychiatry, Psychology and Neuroscience, King's College London, UK; Rebecca MacGregor Legge, Centre for Affective Disorders, Institute of Psychiatry, Psychology and Neuroscience, King's College London, UK; Sarah cohen-Woods, Discipline of Psychiatry, School of Medicine, University of Adelaide, Adelaide, Australia; James H. Cole, Computational, Cognitive and Clinical Adelaide, Australia; James $\mathbf{H}$. Cole, Computational, Cognitive and Clinical
Neuroimaging Laboratory, Department of Medicine, Imperial College London, UK; Katherine J. Aitchison, Department of Psychiatry, University of Alberta, Edmonton Canada; Peter McGuffin, MRC Social, Genetic and Developmental Psychiatry Research Centre, Institute of Psychiatry, Psychology and Neuroscience, King's College London, UK

doi: 10.1192/bjp.207.4.363a

\section{Relevance of ${ }^{123}$ I-FP-CIT SPECT brain scans in routine clinical settings}

The findings from Walker et al's study ${ }^{1}$ do not come as a surprise. The high sensitivity and specificity of ${ }^{123}$ I-ioflupane injection $\left({ }^{123}\right.$ I-FP-CIT) single photon emission computed tomography (SPECT) in diagnosing dementia with Lewy bodies (DLB) relate to a highly selected group of individuals, where underlying vascular pathology, severe mental and physical illness (including delirium) as well as medication interference were excluded. The working group also based their findings on a large group of patients with DLB, in comparison to rather modest size groups previously reported.

How relevant is this study to those of us working in a routine clinical setting? What is the sensitivity and specificity of the ${ }^{123}$ I-FP-CIT SPECT brain scan in differentiating DLB from other dementia syndromes - and pseudodementia in our patients with more advanced age - with polypharmacy, polycomorbidity or recovering from a prolonged spell of acute confusion? Our own clinical experience working with older people with mental and medical health problems suggests that patients can be easily misdiagnosed as having DLB based on their ${ }^{123}$ I-FP-CIT SPECT scans, and this includes individuals with major depression, severe brain trauma accompanied by widespread vascular white matter changes and small vessel disease, HIV encephalopathy, and even an older adult with mild intellectual disability with frontal lobe syndrome and extensive hypoperfusion as demonstrated on the SPECT brain scan. This is another confirmation of the clinician's gullibility when faced with ${ }^{123} \mathrm{I}$-FP-CIT SPECT altered scans, as confirmed by Walker et al. ${ }^{1}$

With the availability of ${ }^{123}$ I-FP-CIT SPECT scans, it is unclear what we have learned from the use of this imaging technique: do we use them for DLB diagnosis - based on their abnormal findings alone - or do we put them in the wider context of our patients' clinical symptomatology and medical history? There is a welldocumented inverse relationship between vascular lesions and Lewy body pathology; ${ }^{2} 30 \%$ of patients with frontotemporal lobe dementia have abnormal scans and a significant reduction in uptake in the putamen and the caudate ${ }^{3}$ (also highlighted by Walker et $a l^{1}$ ). About $5 \%$ of people diagnosed with DLB in fact have vascular dementia ${ }^{4}$ and altered suspected ${ }^{123}$ I-FP-CIT SPECT are also found in Creutzfeldt-Jakob disease. ${ }^{5}$ It is of note that the influence of antipsychotic ${ }^{6}$ and antidepressant medication ${ }^{7}$ in older adults has largely been neglected in research studies in the public domain. The evidence from a limited number of animal ${ }^{8}$ and human ${ }^{9}$ studies clearly indicates that medication (e.g. haloperidol, citalopram, sertraline) reduces ${ }^{123}$ I-FP-CIT dopamine binding to the dopaminergic transporter. However, there is an overwhelming lack of evidence for the most frequently used drugs in the older population, including a number of dopaminergic antagonists, the influence of polypharmacy, the effect of chronic administration of these drugs and modifying effects of advanced age. Until such data are available, it is not surprising that clinicians would be inclined to diagnose and/or accept the diagnosis of DLB based on the evidence of a dopaminergic abnormality. Even in their strictly controlled study, Walker et al ${ }^{1}$ report 5.4\% mismatch between ${ }^{123}$ I-FP-CIT SPECT scan findings and clinical DLB diagnosis. It is now the responsibility of the DLB research community to provide us with further clarification of clinical situations and exclusion criteria when using ${ }^{123}$ I-FP-CIT SPECT scans to diagnose DLB in busy clinical settings.

1 Walker Z, Moreno E, Thomas A, Inglis F, Tabet N, Rainer M, et al. Clinical usefulness of dopamine transporter SPECT imaging with 123I-FP-CIT in patients with possible dementia with Lewy bodies: randomised study. Br J Psychiatry 2015; 206: 145-52.

2 Fukui T, Oowan Y, Yamazaki T, Kinno R. Prevalence and clinical implication of microbleeds in dementia with lewy bodies in comparison with microbleeds in Alzheimer's disease. Dement Geriatr Cogn Dis Extra 2013; 3: 148-60.

3 Morgan S, Kemp P, Booij J, Costa DC, Padayachee S, Lee L, et al. Differentiation of frontotemporal dementia from dementia with Lewy bodies using FP-CIT SPECT. J Neurol Neurosurg PSychiatry. 2012; 83: 1063-70.

4 Kemp PM, Clyde K, Holmes C. Impact of 123I-FP-CIT (DaTSCAN) SPECT on the diagnosis and management of patients with dementia with Lewy bodies: a retrospective study. NuCl Med Commun 2011; 32: 298-302.

5 Ragno M, Scarcella MG, Cacchiò G, Capellari S, Di Marzio F, Parchi P, et al. Striatal [123I] FP-CIT SPECT demonstrates dopaminergic deficit in a sporadic case of Creutzfeldt-Jakob disease. Acta Neurol Scand 2009; 119: 131-4.

6 Barrou Z, Boddaert J, Faucounau V, Habert MO, Greffard S, Dieudonné B, et al. Utility of 123I-FP-CIT SPECT for dementia diagnoses and therapeutic strategies in elderly patients. J Nutr Health Aging 2014; 18: 50-3.

7 Booij J, de Jong J, de Bruin K, Knol R, de Win MM, van Eck-Smit BL. Quantification of striatal dopamine transporters with 123I-FP-CIT SPECT is influenced by the selective serotonin reuptake inhibitor paroxetine: a double-blind, placebo-controlled, crossover study in healthy control subjects. J NuCl Med 2007; 48: 359-66.

8 Nikolaus S, Antke C, Kley K, Beu M, Wirrwar A, Müller HW. Pretreatment with haloperidol reduces (123)I-FP-CIT binding to the dopamine transporter in the rat striatum: an in vivo imaging study with a dedicated small-animal SPECT camera. J Nucl Med 2009; 50: 1147-52.

9 Ziebell M, Holm-Hansen S, Thomsen G, Wagner A, Jensen P, Pinborg LH, et al. Serotonin transporters in dopamine transporter imaging: a head-to-head comparison of dopamine transporter SPECT radioligands 123I-FP-CIT and 123I-PE2I. J NuCl Med 2010; 51: 1885-91. Elizabeta B. Mukaetova-Ladinska, MD, MMedSci, PhD, MRCPsych, Newcastle
Liaison Team for Older Adults, Northumberland, Tyne and Wear NHS Foundation Trust, Newcastle upon Tyne, and Institute for Ageing and Health, Newcastle University, Wolfson Research Centre, Campus for Ageing and Vitality, Newcastle upon Tyne NE4 5PL, UK. Email: Elizabeta.Mukaetova-Ladinska@ncl.ac.uk; Ann Scully, MRCPsych, Newcastle Liaison Team for Older Adults, Northumberland, Tyne and Wear NHS Foundation Trust, Newcastle upon Tyne, UK

doi: $10.1192 /$ bjp.207.4.364

Authors' reply: The data presented were the culmination of a well-designed European multicentre study which adds a valuable data-set on the clinical usefulness of ${ }^{123}$ I-FP-CIT SPECT (DaTSCAN). Although it is correct that the participants in the study were a selected group, as is the case in all clinical trials and similar studies, the sample overall was probably not significantly different in terms of general comorbidities and 
medication to real-world memory clinic patients. It is correct, for example, that vascular pathology and other key comorbidities were excluded but their presence in many instances may point to a different diagnostic pathway. As such, many of these excluded patients would not require a ${ }^{123} \mathrm{I}-\mathrm{FP}-\mathrm{CIT}$ scan to confirm a diagnosis.

We feel that the result of the study is particularly relevant to clinicians in everyday practice. It was not intended as a measure of sensitivity and specificity but rather as a tool of clinical utility in the absence of a recognised gold standard for the diagnosis of DLB. Hence, it is more about the impact on diagnosis than on diagnostic accuracy.

We agree with Mukaetova-Ladinska \& Scully that comorbidities which are prevalent in older people presenting with cognitive impairment add to the diagnostic difficulties. But it is also correct that in these patients specifically, additional information obtained through the ${ }^{123}$ I-FP-CIT scan could prove to be very helpful. For example, the presence of depression and a positive ${ }^{123}$ I-FP-CIT scan may raise the possibility of an emerging DLB picture when other symptoms start to point in this direction. But certainly the ${ }^{123} \mathrm{I}$-FP-CIT scan is not intended as a stand-alone test. First, vascular pathology and structural abnormalities will need to be excluded by magnetic resonance imaging or computed tomography. The ${ }^{123}$ I-FP-CIT scan thereafter can be considered as an adjunct and in no way replaces a full history, cognitive assessment and physical examination. Hence, it is a supporting imaging technique which is considered in the consensus criteria as a suggestive feature. We also agree with Mukaetova-Ladinska \& Scully that frontal lobe symptoms present a further set of diagnostic challenges. However, such symptoms when present could raise the possibility of a frontotemporal dementia and then more appropriate metabolic or perfusion scans may be needed.

It is true that some drugs do alter the overall dopamine transporter uptake, but in practice, few of these will have an impact on visual analysis of ${ }^{123}$ I-FP-CIT scan images and in reality only a few drugs need to be withdrawn. Of the drugs mentioned for example, haloperidol does block postsynaptic $\mathrm{D}_{2}$ receptors but not the dopamine transporter and therefore should not affect uptake. We do believe, however, that more studies are still needed to clarify this issue. Likewise, more data are still needed to support further the clinical utility of the ${ }^{123}$ I-FP-CIT scan investigation and outcomes.

Zuzana Walker, MD, FRCPsych, Division of Psychiatry, University College London, and North Essex Partnership University NHS Foundation Trust, St Margaret's Hospital The Plain, Epping, Essex CM16 6TN, UK. Email: z.walker@ucl.ac.uk; Naji Tabet, MD, MRCPsych, Postgraduate Medicine, Brighton and Sussex Medical School, Brighton, UK

doi: $10.1192 / \mathrm{bjp} .207 .4 .364 \mathrm{a}$

\section{Correction}

Reciprocal associations between smoking cessation and depression in older smokers: findings from the English Longitudinal Study of Ageing. BJPsych, 207, 243-249. The following funding acknowledgement was omitted:

The study was in part funded by Greenwich University, Cancer Research UK and the Economic and Social Research Council. The funders had no role in the study design; in the collection, analysis and interpretation of data; in writing of the report; or in the decision to submit the paper for publication.

Consequently, usage of the published paper is governed by the Creative Commons Attribution (CC BY) licence.

The online version of this paper has been corrected postpublication, in deviation from print and in accordance with this correction.

doi: 10.1192/bjp.207.4.365 